\title{
Neural network-based anomalous diffusion parameter estimation approaches for Gaussian processes
}

\author{
Dawid Szarek ${ }^{1}$ (D)
}

Accepted: 29 July 2021 / Published online: 24 August 2021

(C) The Author(s) 2021

\begin{abstract}
Anomalous diffusion behavior can be observed in many single-particle (contained in crowded environments) tracking experimental data. Numerous models can be used to describe such data. In this paper, we focus on two common processes: fractional Brownian motion (fBm) and scaled Brownian motion (sBm). We proposed novel methods for sBm anomalous diffusion parameter estimation based on the autocovariance function (ACVF). Such a function, for centered Gaussian processes, allows its unique identification. The first estimation method is based solely on theoretical calculations, and the other one additionally utilizes neural networks (NN) to achieve a more robust and well-performing estimator. Both $\mathrm{fBm}$ and $\mathrm{sBm}$ methods were compared between the theoretical estimators and the ones utilizing artificial NN. For the NN-based approaches, we used such architectures as multilayer perceptron (MLP) and long short-term memory (LSTM). Furthermore, the analysis of the additive noise influence on the estimators' quality was conducted for NN models with and without the regularization method.
\end{abstract}

Keywords Anomalous diffusion .

Fractional Brownian motion - Scaled Brownian motion ·

Neural network · Deep learning $\cdot$ LSTM

Mathematics Subject Classification 60G15 - 60G18 . $62 \mathrm{M} 10 \cdot 68 \mathrm{~T} 07$

Dawid Szarek

234856@student.pwr.edu.pl

1 Faculty of Pure and Applied Mathematics, Hugo Steinhaus Center, Wroclaw University of Science and Technology, Wybrzeże Wyspiańskiego 27, 50-370 Wroclaw, Poland

\section{Introduction}

The anomalous diffusion behavior can be observed in various environments starting from single-particle motion in crowded environments [1-6], to finance [7, 8], ecology [9], hydrology [10], biology [11, 12] as well as meteorology and geophysics [13, 14]. The most commonly used definition of anomalous diffusion is one using the second moment of the process $\{X(t)\}$ :

$\mathbb{E}\left[X^{2}(t)\right] \sim t^{\alpha}$,

which, for processes starting at 0 , is also known under the name of mean square displacement (MSD) [15]. Standard examples of the anomalous diffusion processes are fractional Brownian motion [13, 16] (fBm), scaled Brownian motion [17-19], Lévy stable motion [20], continuous-time random walk [21, 22], and subordinated processes (also called time-changed processes) [23-30]. In this paper, we focus on the first two examples (fBm and sBm) described in detail in the following Sect. 2 .

In real data analysis, the main difficulty is fitting the model and estimating its parameters. While both fBm and sBm are simple models, there are problems with reliable estimation using classic methods, especially when the measurement methods of the trajectories are inaccurate [31]. We observe that classical approaches degrade severely when the scale of the noise is substantial up to the point when they have a problem with the distinction between sub- and superdiffusion.

Here, we focus on the methods utilizing sample autocovariance function (ACVF) [32]; however, other approaches are viable [33]. It is motivated by the theoretical properties of this function, namely that for a centered Gaussian process only ACVF is needed for unique identification. We compare the classical approaches with the 
proposed NN-based ones and analyze the influence of the added noise on the estimation quality.

The rest of the paper has the following structure. First, we define normal and anomalous diffusion using partial differential equations and the stochastic processes emerging from their solutions with the focus on $\mathrm{fBm}$ and $\mathrm{sBm}$. In the third section, the estimation methods are presented, starting from the summary of the fBm identification approach (described in detail in [34]) ending with the $\mathrm{sBm}$ identification methodology and the description of NN architecture. In the following section, the methods' evaluation and comparison occur. The data generation methodology is presented with the error analysis for both data with and without the noise of different scales. We also inspect the influence of adding noise to the training samples. The last section is a summary of the paper with propositions for further improvement of the presented methodology.

\section{Anomalous diffusion models}

In this section, we describe normal and anomalous diffusion. We also define and characterize two examples of such processes, namely the $\mathrm{fBm}$ and $\mathrm{sBm}$ processes.

The roots of diffusion and anomalous diffusion are in physics; for this reason, we will define it using the partial differential equation (PDE) [35]:

$\frac{\partial p}{\partial t}=D \Delta p$

where $p(x, t)$ is density of a material in time $t$ and point in space $x, D$ is a constant determining the rate of dispersion in the volume and $\Delta$ is Laplace operator defined as $\Delta=\nabla^{2}=\sum_{i=1}^{d} \frac{\partial^{2}}{\partial^{2} x_{i}}$. The solution of the equation (2) (under certain assumptions) leads to the probability density function (PDF) of Gaussian distribution [35]:

$p(x, t)=\frac{1}{\sqrt{4 \pi D t}} e^{-\frac{x^{2}}{4 D t}}$.

Thus, normal diffusion can be modeled using Brownian motion [36].

However, when using this model, we assume that in the system there are no external forces and it is in equilibrium. Furthermore, the diffusivity coefficient has to be constant. For those reasons, a generalization was proposed, namely anomalous diffusion. As mentioned in the introduction, it is defined using ensemble MSD (1). For Brownian motion $\{B(t)\}$ (normal diffusion) $\mathbb{E}\left[B^{2}(t)\right] \propto t$ thus any deviation of the second moment from the linearity is called anomalous. For $\alpha=1$, as already established, the process is called normal diffusion. When $\alpha<1$ the process is subdiffusive and for $\alpha>1$ superdiffusive.

There are many examples of anomalous diffusion processes, among which fractional Brownian motion ( $\mathrm{fBm})$ $[13,16]$ and scaled Brownian motion (sBm) [17-19] are the most popular ones.

\subsection{Fractional Brownian motion}

One possible generalization of diffusion equation (2) is to include the diffusivity that is nonconstant. The $\mathrm{fBm}$ comes from such an extension, where $D$ is replaced by function $D(t):=\alpha C t^{\alpha-1}$ to get the following PDE [37]:

$\frac{\partial p}{\partial t}=\alpha C t^{\alpha-1} \Delta x=D(t) \Delta x, \quad \alpha \in(0,2)$.

The solution to this equation can also be associated with a Gaussian process as:

$p(x, t ; \alpha)=\frac{1}{\sqrt{4 \pi C t^{\alpha}}} e^{-\frac{x^{2}}{4 C t^{\alpha}}}$.

Comparing Eq. (3) to Eq. (4) one can observe that for $\alpha=$ 1 the solutions are alike.

The $\mathrm{fBm}\left\{B_{\alpha}(t)\right\}, \alpha \in(0,2)$ is defined as a Gaussian process $B_{\alpha}(t) \sim \mathcal{N}\left(0,2 C t^{\alpha}\right)$ with the following characteristics [38]:

$\mathbb{E}\left[B_{\alpha}(t)\right]=0 \quad \mathbb{E}\left[B_{\alpha}(s) B_{\alpha}(t)\right]=C\left(t^{\alpha}+s^{\alpha}-|t-s|^{\alpha}\right)$.

The process is self-similar $B_{\alpha}(a t) \sim a^{\alpha / 2} B_{\alpha}(t)$ with stationary increments $B_{\alpha}(t+\tau)-B_{\alpha}(t) \sim B_{\alpha}(\tau), \tau>0$. For $\alpha>1$ the process exhibits the so-called long-range dependence $\sum_{n=1}^{\infty} \mathbb{E}\left[B_{\alpha}(1)\left(B_{\alpha}(n+1)-B_{\alpha}(n)\right)\right]=\infty$.

\subsection{Fractional Brownian noise}

Fractional Brownian noise (fBn) is defined as the increment process of $\mathrm{fBm}$, namely:

$$
\Delta B_{\alpha}(t)=B_{\alpha}(t)-B_{\alpha}(t-1), \quad t \in \mathbb{N}_{+} .
$$

As it was established in Sect. 2.1, fBm has stationary increments and thus $\mathrm{fBn}$ is (weakly) stationary, which implies that the distribution of the process does not change in time $\Delta B_{\alpha}(t) \sim B_{\alpha}(1) \sim \mathcal{N}(0, C)$. Furthermore, the ACVF only depends on the lag $\tau$ :

$$
\begin{aligned}
& \mathbb{E}\left[\Delta B_{\alpha}(t+\tau) \Delta B_{\alpha}(t)\right] \\
& \quad=C\left(|\tau-1|^{\alpha}+|\tau+1|^{\alpha}-2 \tau^{\alpha}\right), \quad t, \tau \in \mathbb{N} .
\end{aligned}
$$

This crucial property of fBn allows for a feasible estimation of the $\alpha$ parameter as the estimation procedure can rely only on the ACVF evaluated for certain lags $\tau$. 


\subsection{Scaled Brownian motion}

Scaled Brownian motion process is a simple modification of the Brownian motion $\{B(t)\}$. Namely, it is constructed from the ordinary Brownian motion by "scaling" time:

$\widetilde{B}_{\alpha}(t)=B\left(t^{\alpha}\right)$.

Many properties of $\mathrm{sBm}$ are shared with standard Brownian motion. What is more, for $\alpha=1$, the $\left\{\widetilde{B}_{\alpha}(t)\right\}$ reduces to regular Brownian motion $\{B(t)\}$. The process is Gaussian $\widetilde{B}_{\alpha}(t) \sim \mathcal{N}\left(0,2 D t^{\alpha}\right)$ and self-similar $B\left((a t)^{\alpha}\right) \sim a^{\alpha / 2} B_{\alpha}\left(t^{\alpha}\right)$. Furthermore, it does have independent yet not stationary increments:

$B\left((t+\tau)^{\alpha}\right)-B\left(t^{\alpha}\right) \sim B\left((t+\tau)^{\alpha}-t^{\alpha}\right) \nsim B\left(\tau^{\alpha}\right), \quad \tau>0, \alpha \neq 1$.

As the process is Gaussian, it can be fully characterized by the mean value and covariance matrix:

$\mathbb{E}\left[\widetilde{\boldsymbol{B}}_{\alpha}(t)\right]=0 \quad \mathbb{E}\left[\widetilde{\boldsymbol{B}}_{\alpha}(s) \widetilde{\boldsymbol{B}}_{\alpha}(t)\right]=2 D \min \left\{t^{\alpha}, s^{\alpha}\right\}$.

\subsection{Scaled Brownian noise}

Similarly to fBn (Sect. 2.2), sBn is defined by the differentiation of sBm:

$\Delta \widetilde{B}_{\alpha}(t)=\widetilde{B}_{\alpha}(t)-\widetilde{B}_{\alpha}(t-1), \quad t \in \mathbb{N}_{+}$.

This process, alike $\mathrm{fBn}$, has the expected value equal to zero $\mathbb{E} \Delta \widetilde{B}_{\alpha}(t)=0$. However, the ACVF is still dependent on both $t$ and $\tau$ [17]:

$\mathbb{E}\left[\Delta \widetilde{B}_{\alpha}(t+\tau) \Delta \widetilde{B}_{\alpha}(t)\right]=2 D \alpha t^{\alpha-1} \mathbb{1}_{\tau=0}$,

where $\mathbb{1}_{\tau=0}$ takes the value of 1 when $\tau=0$ and 0 otherwise. Furthermore, the distribution of the process is as follows:

$$
\Delta \widetilde{B}_{\alpha}(t) \sim \mathcal{N}\left(0,2 D \alpha t^{\alpha-1}\right) .
$$

\section{Methodology}

In this section, the methodology to estimate the $\alpha$ parameter (the coefficient responsible for the type of diffusion) for $\mathrm{fBm}$ and $\mathrm{sBm}$ will be presented. The proposed approach relies on the properties of Gaussian processes, namely that those can be uniquely identified with the expected value $\boldsymbol{\mu}$ and covariance matrix $\boldsymbol{\Sigma}$ [39]:

$\boldsymbol{\mu}=\mathbb{E}\{X(t)\} \quad$ and $\quad \boldsymbol{\Sigma}=\operatorname{Cov}\{X(t)\}=\mathbb{E}\left[\{X(t)\}\{X(t)\}^{\top}\right]-\boldsymbol{\mu} \boldsymbol{\mu}^{\top}$,

where $\{X(t)\}^{\top}$ denotes transposition of the column vector $\{X(t)\}$.

Let us consider $N$ trajectories of a Gaussian process $\{X(t)\}$ where $t \in\{0, \ldots, T\}$ with $X(0)=0$ almost surely.
If the mean $\boldsymbol{\mu}$ is equal to zero, as it is in $\mathrm{fBm}$ and $\mathrm{sBm}$, we can consider just the covariance matrix $\boldsymbol{\Sigma}$. However, it has many values, among which many might not provide new information, as the trajectory has length $T+1$ and covariance matrix has size $(T+1)^{2}$. Furthermore, one trajectory $(N=1)$ is insufficient to calculate a reliable estimate, as the estimator of $\boldsymbol{\Sigma}$ for a process $\{X(t)\}$ is as follows:

$$
\hat{\boldsymbol{\Sigma}}_{t, s}=\frac{1}{N} \sum_{n=1}^{N} X_{n}(t) X_{n}(s)
$$

\subsection{Fractional Brownian motion}

As it is shown in Sect. 2.1, the fBm covariance depends on both parameters $t$ and $s$ (Eq. (5)). This problem can be simply resolved by differentiating the process to get $\mathrm{fBn}$ (see Sect. 2.2). Then, the covariance depends on the difference $|t-s|=\tau$ (see ACVF in Eq. (6)). Such ACVF can be efficiently estimated from a single trajectory using the following formula:

$$
\hat{\boldsymbol{\Sigma}}_{\tau}=\frac{1}{T} \sum_{t=1}^{T-\tau} \Delta B_{\alpha}(t+\tau) \Delta B_{\alpha}(t)
$$

Furthermore, for the ACVF to be independent of the scale parameter $C$, we can simply scale the trajectory: $\left\{\frac{\Delta B_{\alpha}(t)}{\sqrt{\hat{\mathbf{\Sigma}}_{\tau=0}}}\right\}$ as $\hat{\boldsymbol{\Sigma}}_{\tau=0} \stackrel{T \rightarrow \infty}{\longrightarrow} C$. Then, the ACVF vector

$$
\left\{\frac{\hat{\boldsymbol{\Sigma}}_{\tau}}{\hat{\boldsymbol{\Sigma}}_{0}}\right\}_{\tau=1}^{T}
$$

can be used to estimate $\alpha$. Details are described in the following article [34].

\subsection{Scaled Brownian motion}

However, for sBm, the sBn's ACVF does not depend only on a lag $\tau$, thus it is needed to approach the problem differently. The idea is to make the vector stationary and as stable numerically as possible.

Using Eq. (7), one can obtain the following results:

$$
\frac{\mathbb{E}\left[\left(\Delta \widetilde{B}_{\alpha}(t)\right)^{2}\right]}{\mathbb{E}\left[\left(\Delta \widetilde{B}_{\alpha}(1)\right)^{2}\right]}=t^{\alpha-1} \Rightarrow \alpha=\frac{\log \left(\frac{\mathbb{E}\left[\left(\Delta \widetilde{B}_{\alpha}(t)\right)^{2}\right]}{\mathbb{E}\left[\left(\Delta \widetilde{B}_{\alpha}(1)\right)^{2}\right]}\right)}{\log (t)}+1 .
$$

While the goal is to estimate $\alpha$ using one trajectory, Eq.(9) is used as the suggestion on how to "stationarize" the 
process. Hence, the following process will be used for estimation of $\alpha$ parameter:

$$
\{\hat{\xi}(t)\}_{t=2}^{T}=\left\{\frac{\log \left[\left(\frac{\Delta \widetilde{B}_{\alpha}(t)}{\Delta \widetilde{B}_{\alpha}(1)}\right)^{2}\right]}{\log (t)}\right\}_{t=2}^{T} .
$$

Similar calculations could be conducted for sBm, however, the resulting estimators characterize a large variance causing numerical instability.

Omission of the expectations certainly changes the behavior of the vector. However, as we show, this estimator is unbiased for each value $t$.

Lemma 1 For each $t \in\{2,3, \ldots, T\}$, the estimators $\{\hat{\xi}(t)\}$ defined in Eq. (10) are unbiased.

Proof To show that the estimators are unbiased, it is needed to validate that the expectation matches the estimated parameter, namely:

$\mathbb{E}[\hat{\xi}(t)]=\mathbb{E}\left[\frac{\log \left(\left(\frac{\Delta \widetilde{B}_{\alpha}(t)}{\Delta \widetilde{B}_{\alpha}(1)}\right)^{2}\right)}{\log (t)}\right]=\alpha-1$.

Using Jensen's inequality [40] and Cauchy-Schwarz inequality [41], we can show that:

$$
\begin{aligned}
\mathbb{E}\left[\frac{\log \left(\left(\frac{\Delta \widetilde{B}_{\alpha}(t)}{\Delta \widetilde{B}_{\alpha}(1)}\right)^{2}\right)}{\log (t)}\right] & \leq \frac{2 \log \left(\mathbb{E}\left[\frac{\Delta \widetilde{B}_{\alpha}(t)}{\Delta \vec{B}_{\alpha}(1)}\right]\right)}{\log (t)} \\
& =\frac{\log \left(\left(\mathbb{E}\left[\frac{\Delta \widetilde{B}_{\alpha}(t)}{\Delta \widetilde{B}_{\alpha}(1)}\right]\right)^{2}\right)}{\log (t)} \\
& \leq \frac{\log \left(\frac{\mathbb{E}\left[\left(\Delta \widetilde{B}_{\alpha}(t)\right)^{2}\right]}{\mathbb{E}\left[\left(\Delta \widetilde{B}_{\alpha}(1)\right)^{2}\right]}\right)}{\log (t)}=\alpha-1
\end{aligned}
$$

and

$$
\begin{aligned}
\mathbb{E}\left[\frac{\log \left(\left(\frac{\Delta \widetilde{B}_{\alpha}(t)}{\Delta \widetilde{B}_{\alpha}(1)}\right)^{2}\right)}{\log (t)}\right] & \geq-\frac{2 \log \left(\mathbb{E}\left[\frac{\Delta \widetilde{B}_{\alpha}(1)}{\Delta \widetilde{B}_{\alpha}(t)}\right]\right)}{\log (t)} \\
& =-\frac{\log \left(\left(\mathbb{E}\left[\frac{\Delta \widetilde{\Delta B}_{\alpha}(1)}{\Delta \widetilde{B}_{\alpha}(t)}\right]\right)^{2}\right)}{\log (t)} \\
& \geq-\frac{\log \left(\frac{\mathbb{E}\left[\left(\Delta \widetilde{B}_{\alpha}(1)\right)^{2}\right]}{\mathbb{E}\left[\left(\Delta \widetilde{B}_{\alpha}(t)\right)^{2}\right]}\right)}{\log (t)}=\alpha-1 .
\end{aligned}
$$

Consequently, this estimator is unbiased:

$$
\mathbb{E}[\hat{\xi}(t)]=\alpha-1 \text {. }
$$

One could think that with such a property, it should be reasonable for a single trajectory to define the following unbiased estimator:

$$
\hat{\alpha}:=1+\frac{1}{T-2} \sum_{t=2}^{T} \hat{\xi}(t) .
$$

However, it characterizes the large variance.

In Fig. 1, sample trajectories of sBm with corresponding evaluations of $\{\hat{\xi}(t)\}$ statistics (10) are presented. The distribution of $\{\hat{\xi}(t)\}$ from certain $t$ starts to stabilize. It is easily observed that the distribution is not symmetric-it is highly skewed. In addition, the moving average does not necessarily match the true value $\xi(t)=\alpha-1$.

In Fig. 2, we see that although the estimator's mean matches the true value, yet the spread is severe. Depending on the trajectory size, the observed mean absolute error (MAE) for $T=1024$ is MAE $=1.030$ and for smaller sample $T=32$ it reaches MAE $=1.255$. Thus, while using this estimator, it is highly probable to confuse the type of anomalous diffusion. Consequently, a superior aggregation function is needed.

\subsection{Neural network algorithm}

The architecture of the proposed neural network will be simple and similar to one in [34]. However, one significant change will be done that allows for variable input vector size. The first layer will be exchanged for a recurrent layer, to be precise, the long short-term memory (LSTM) $[42,43]$. The ingestion of the input vector $\{\hat{\xi}(t)\}$ will be done in the natural order-with increasing time. It will 


\section{$\alpha=0.25$}
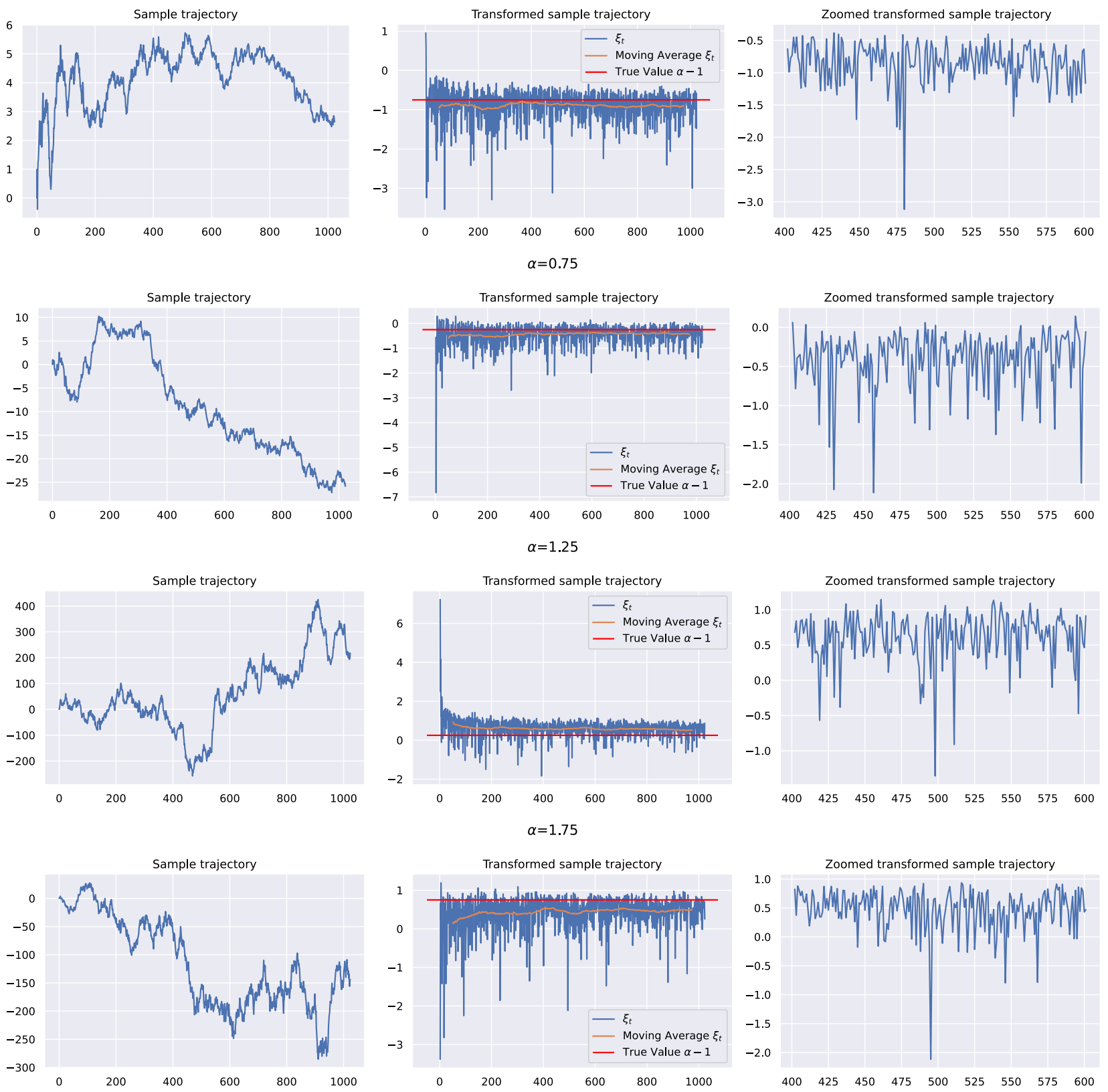

Fig. 1 The figures in the second column present the transformed (Eq. (10)) sBm trajectories $(T=1024)$ from first column. The last column presents close-up on the transformed trajectory to show details of the estimates

allow the algorithm to disregard the more variable part (beginning) of the $\{\hat{\xi}(t)\}$ vector (see Fig. 1) and learn more stably. In recurrent neural networks (RNNs), the so-called exploding and vanishing gradient problem occurs [44], and this approach minimizes the risk of such an event occurring.

The RNN architecture is especially needed because with multilayer perceptron (MLP) [45] (used in [34]) the model's error does not plateau (Fig. 3)-with increasing $\widetilde{T}$ the error is decreasing with log-linear rate (from certain $\widetilde{T}$ ).
This is inline with the law of large numbers [46]. Furthermore, we observe that addition of the mean does improve the performance at first. However, from certain $\widetilde{T}$, the difference is negligible, despite the fact that there is the difference of $\min _{\widetilde{T}}(T-\widetilde{T})=512$ values used for estimation (as $T=1024$ ).

Thus, the proposed model is built from the LSTM layer of size 128 followed by 5 densely connected layers (with the corresponding number of neurons $128,128,128,64$, 
Fig. 2 Boxplots of $\hat{\alpha}$ for $\mathrm{sBm}$ trajectories of length $T=1024$ with 1000 Monte Carlo simulations for each $\alpha \in\{0.25,0.5, \ldots, 1.75\}$.

Yellow bar represents true value $\alpha$ and white dot observed mean

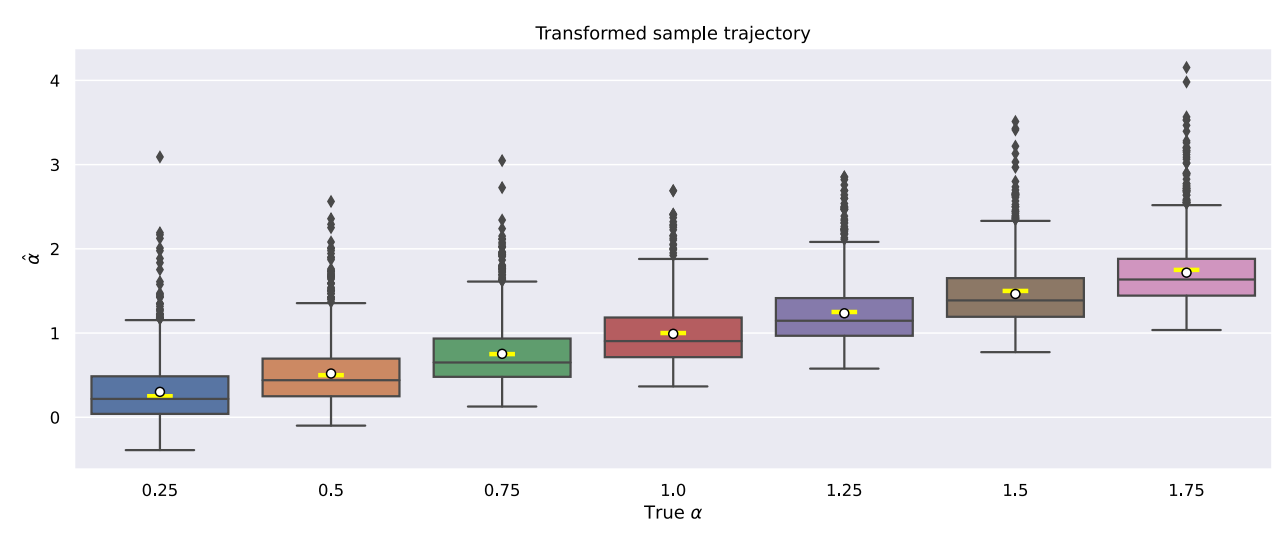

Fig. 3 MAE for MLP models. Model "Without mean" represents a model for which we take $\widetilde{T}$ (see Sect. 4.1 for details) first values of $\{\hat{\xi}(t)\}$ and model "With mean" has last value of input vector exchanged for mean of $\{\hat{\xi}(t)\}_{t=\widetilde{T}}^{T}$
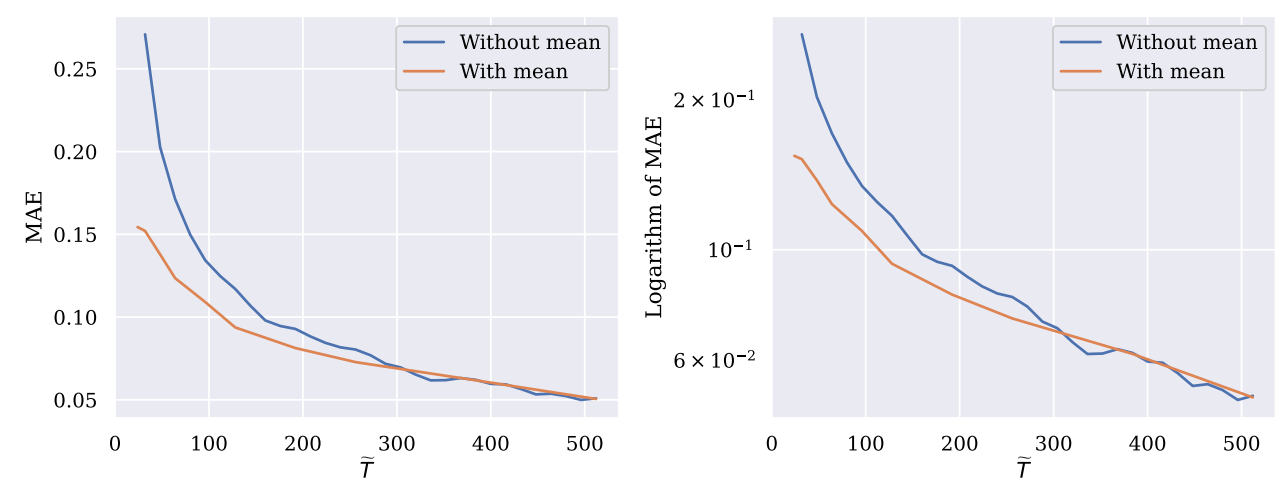

64, 32) with SiLU [47] activation function (also known as Swish-1 [48]):

$a(x)=x \cdot \sigma(x)=\frac{x}{1-e^{-x}}$.

However, other well-performing functions could be used like GeLU [49] (very similar to SiLU), ELU [50] or classical Leaky ReLU [51]. The choice of SiLU was dictated by its superior performance in the majority of NN applications. The output layer consists of 1 neuron corresponding to estimated value $\alpha$. To constrain the NN to estimate values from the interval $(0,2)$, the scaled sigmoid $\widetilde{\sigma}(x)$ activation function was used:

$$
\widetilde{\sigma}(x)=2 \cdot \sigma(x)=\frac{2}{1-e^{-x}} .
$$

To learn the NN's parameters, Adam optimization algorithm [52] was applied as it quickly converges to a minimum of a loss function [53-56]. The optimization problem is to minimize mean square error (MSE) loss function [45]:

$L(\alpha, \hat{\xi})=\frac{1}{N} \sum_{i=1}^{N}\left(\alpha_{i}-\mathrm{NN}\left(\hat{\xi}_{i}\right)\right)^{2}$

The optimization was set to a maximum of 50 epochs (with early stopping when the validation error does not improve for 5 epochs). Furthermore, the learning rate was set to decrease when the validation error plateaus (by a factor of 10 starting from 0.01 ).

While in [34] MLP NN architecture was used for intelligent $\mathrm{fBm}$ identification, we will attach such architecture into plots.

\section{Monte Carlo simulation study}

The classic estimators, proposed (for $\mathrm{sBm}$ ) in Sect. 3.2 and (for $\mathrm{fBm}$ ) in article [34], assume a certain model and with that information, the comparison of the theoretical ACVF to the true one is made. On the other hand, the NN-based methods propose a certain structure of a mapping that is to be derived from a dataset. The universal approximation theorem [57] implies that any function can be approximated by a neural network with a single hidden layer and sigmoid $\sigma(x)$ activation function (12). Thus, the proposed model (in Sect. 3.3), to be viable, needs to learn from the data generated by the theoretical process. For this reason, we first present the method for data generation. To make the comparison easier, the models are of the same size (layer and parameter count). Then, we compare each method using a prediction error measure-MAE. Furthermore, we check each methods' resilience to noise (which simulates the error of the measurement equipment [31]). 


\subsection{Data generation}

Standard machine learning approaches use the division of the data into 3 distinct subsets: training, validation, and test set. This step is important as the NNs are prone to overfitting (remembering the data). To measure whenever the trained model suffers from this effect, a validation set is used. This set is also used to fine-tune the hyperparameters of the model. The final performance of the model is measured using the test set.

We generate the datasets with 6 lengths of trajectories $T \in\left\{2^{5}, 2^{6}, \ldots, 2^{10}\right\}$ for models to learn the "noise" caused by the small sample size and the more stable true values of the ACVF estimates (law of large numbers). For a single trajectory length, we generated $N_{\text {train }}=262144$ $\left(2^{18}\right), N_{\text {val }}=32768\left(2^{15}\right)$, and $N_{\text {test }}=8192\left(2^{13}\right)$ trajectories for training, validation, and test set, accordingly. Each trajectory is generated with a random $\alpha$ parameter taken from the uniform distribution $\mathcal{U}(0,2)$. The motivation behind such sampling is for the models to learn all possible cases of anomalous diffusion evenly.

For the LSTM NN model (defined in Sect. 3.3), we create an input vector of random size, namely we put $\widetilde{T}=\mathcal{D U}(32, T)$. It is to force the NN to learn a more robust and efficient estimator. The choice of 32 as a lower limit of $\widetilde{T}$ parameter is caused by the fact that for $\mathrm{fBm}$ it is the minimal number of ACVF lags needed for the estimation error to plateau (as shown in [34]). Thus, for the comparison, the same strategy will be applied for sBm.

For the noise influence study, we simulate the measurement error by the addition of independent, identically distributed (i.i.d.) centered Gaussian noise $\mathcal{N}\left(0, \sigma^{2}\right)$ to raw trajectories.

\subsection{Methods' comparison}

Here, we compare the "Naive", MLP and LSTM methods for two models ( $\mathrm{fBm}$ and $\mathrm{sBm}$ ). The "Naive" method for $\mathrm{fBm}$ (as described in [34]) is given by the formula:

$\hat{\alpha}_{\text {Naive-fBm }}=\log _{2}\left(2 \hat{\Sigma}_{1}+2\right)$

and the estimator for $\mathrm{sBm}$ is given in Eq. (11).

In Fig. 4, for each process and method, quantile lines are presented. We observe that the "Naive" method for $\mathrm{fBm}$ behaves vastly better than the one for $\mathrm{sBm}$. It has low accuracy across all the $\alpha$ parameter space. The median of absolute error (AE) is always higher than 1. Consequently, even for edge cases of anomalous diffusion, with this estimator, half of the trajectories' diffusion type will be misclassified. The performance is greatly improved with the NN approach. The upper quantile does not even reach the error of 1 . Indicating that the method is much more robust. However, comparing the same models for different processes, there is a significant difference in the accuracy with which those methods estimate $\alpha$. NN's MAE for fBm is on the level of $\mathrm{MAE}_{\mathrm{fBm}}=0.06$ when for $\mathrm{sBm}$ it is almost of order of magnitude greater $\mathrm{MAE}_{\mathrm{SBm}}=0.21$. This is undoubtedly caused by the fact that fBm's ACVF provides more aggregated and essential information about the process. While for $\mathrm{sBm}$, the information is much more spread across the whole vector. This effect could also be observed when analyzing the influence of input vector size on the performance (Fig. 3). Furthermore, for both fBm and sBm, MLP and LSTM models perform similarly; thus, in real-life applications, when the vectors are of constant size (or differ by a small margin), it is recommended to opt for a simpler and faster approach, namely the MLP.

When analyzing the influence of the added noise on estimators, in Fig. 5, one can observe that all models suffer. The smallest change is apparent in sBm's "Naive" method, as it in a noiseless environment struggled to estimate correctly. After the addition of noise, the variance of the estimator increased and the median stabilized at $\mathrm{MAE}=1$. Furthermore, $\mathrm{NN}$ estimators for $\mathrm{sBm}$ decreased their accuracy significantly even for small $\sigma$. However, the level of MAE is fairly constant for even larger amounts of noise (large $\sigma$ ). The NN-based estimators for fBm are much more resilient to noise as they steadily increase their MAE with increasing $\sigma$. However, they reach higher levels than $\mathrm{sBm}$, where the type of diffusion is hard to distinguish. For $\mathrm{fBm}$, the $75 \%$ quantile almost reaches $\mathrm{AE}_{\mathrm{fBm}}=1$ while for $\mathrm{sBm}$ the same quantile only reaches $\mathrm{AE}_{\mathrm{sBm}}=0.5$.

Nevertheless, for $\mathrm{NN}$, it is fairly simple to add the assumption of noise to the model. As it trains from trajectories, thus, all is needed is to augment the dataset by adding the Gaussian noise to the trajectories. Then, the NN should become more resistant to the noise. This technique is also known as dataset augmentation or model regularization [58].

In Fig. 6, accuracy metrics for the retrained models are presented. $\mathrm{fBm}$ model has learned a significantly more resilient estimator as the error only starts to increase around $\sigma=1.5$. One cannot say the same about the retrained sBm $\alpha$ estimator as Fig. $6 \mathrm{~b}$ greatly resembles results for the previously presented model (Fig. 5b). It is more vivid in Fig. 7, where Figs. 5 and 6 are overlaid. There, one can see that for both processes, the models, at the expense of the noiseless case, improved the distribution of the error for environments with a larger noise. For $\mathrm{fBm}$ the change is evident, however, for $\mathrm{sBm}$ it is more subtle. The median error did not change significantly, yet the distribution is more concentrated - the upper quantiles are considerably lower. Consequently, the issue of discerning the diffusion type is reduced. 

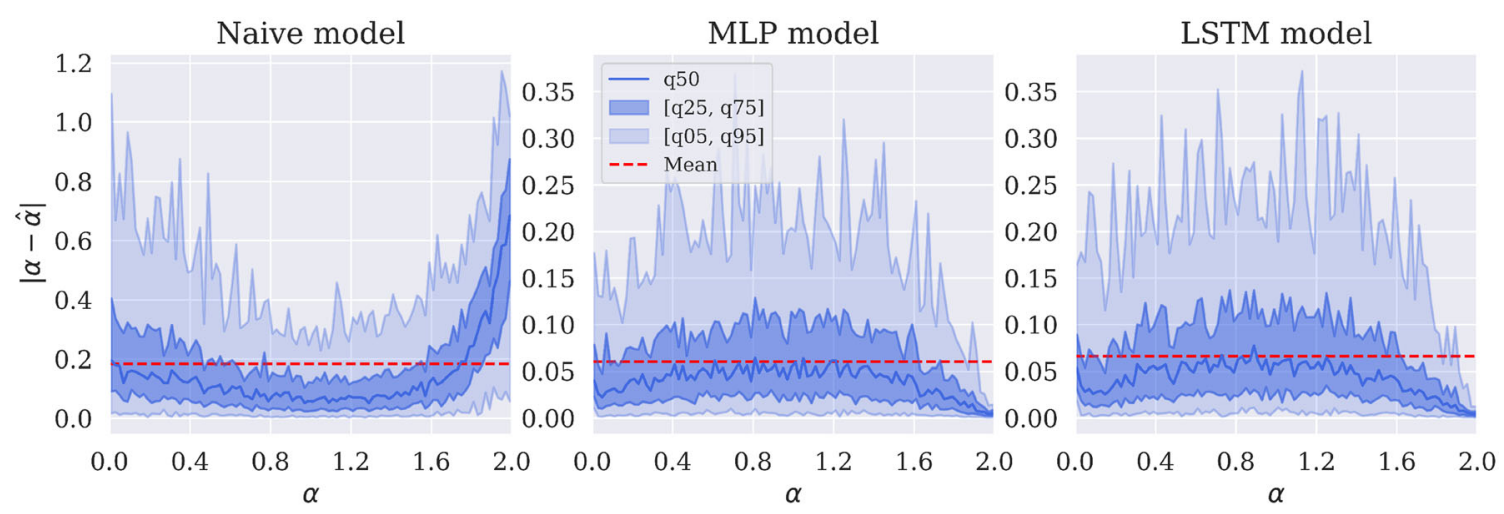

(a) MAE of $\alpha$ parameter estimating models for fBm anomalous diffusion process.
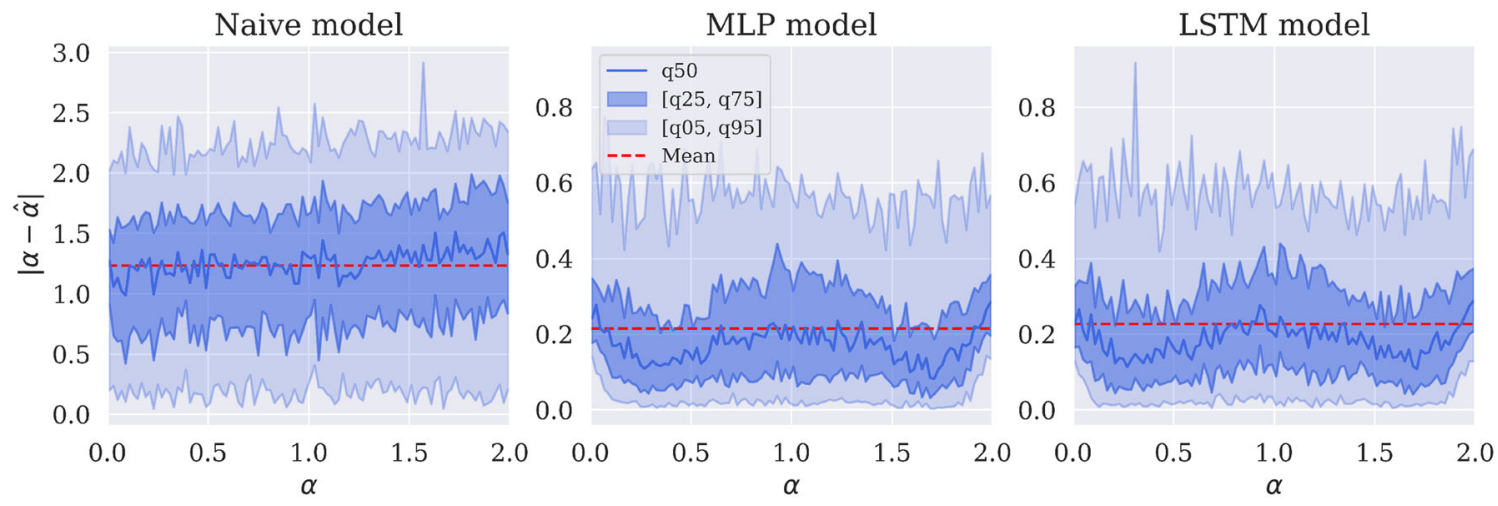

(b) MAE of $\alpha$ parameter estimating models for sBm anomalous diffusion process.

Fig. 4 Figures present MAE for fBm and sBm. Each plot contains quantile lines across true parameter space $\alpha \in(0,2)$. This allows to easily compare models for different regimes of anomalous diffusion. The scale of y axis is shared only between second and third figures

This vastly different behavior of the models could be reasoned by the fact that $\mathrm{sBm}$, because of independent increments, can utilize only $\tau=0$ lag. While the added noise is independent, it influences solely $\tau=0$ ACVF's lag. For this reason, it is much harder to separate the effect of noise in the approach proposed for the sBm process. For $\mathrm{fBm}$, the problem is much simpler to handle because there are other lags $\tau \neq 0$ available which values are not obscured by the noise.

\section{Summary and conclusions}

Anomalous diffusion phenomenon may emerge in various applications. For this reason, we explored two popular anomalous diffusion Gaussian processes used to model such behavior. For both processes, although similar, we presented different methodologies to identify the $\mathrm{fBm}$ and $\mathrm{sBm}$. Even though we used those specific processes, it could be any Gaussian process that is either weakly stationary or with independent increments. Showing the universality of those methods.

The methodology is based on the crucial property of Gaussian processes, namely that such a process can be uniquely identified by the mean vector and covariance matrix. Consequently, when trying to design features describing the process, we can solely base on those quantities. For centered, weakly stationary processes (such as $\mathrm{fBn}$ ), we can depend exclusively on ACVF which can be efficiently estimated from a single trajectory. The approach in [34] utilizes such ACVF vectors to identify fBm.

In our novel approach, concerning the estimation of anomalous diffusion parameter $\alpha$ for $\mathrm{sBm}$, we also utilize ACVF; however, as $\mathrm{sBn}$ is nonstationary and independent, the same method could not be used. Thus, we proposed a way to "stationarize" process (with the help of ACVF), which has proven to give reasonably stable trajectories. This allowed us to feasibly estimate the $\alpha$ parameter. Although the standard approach to create an estimator has failed because of the large variance, the NN-based one has produced an estimator which allows estimating the 


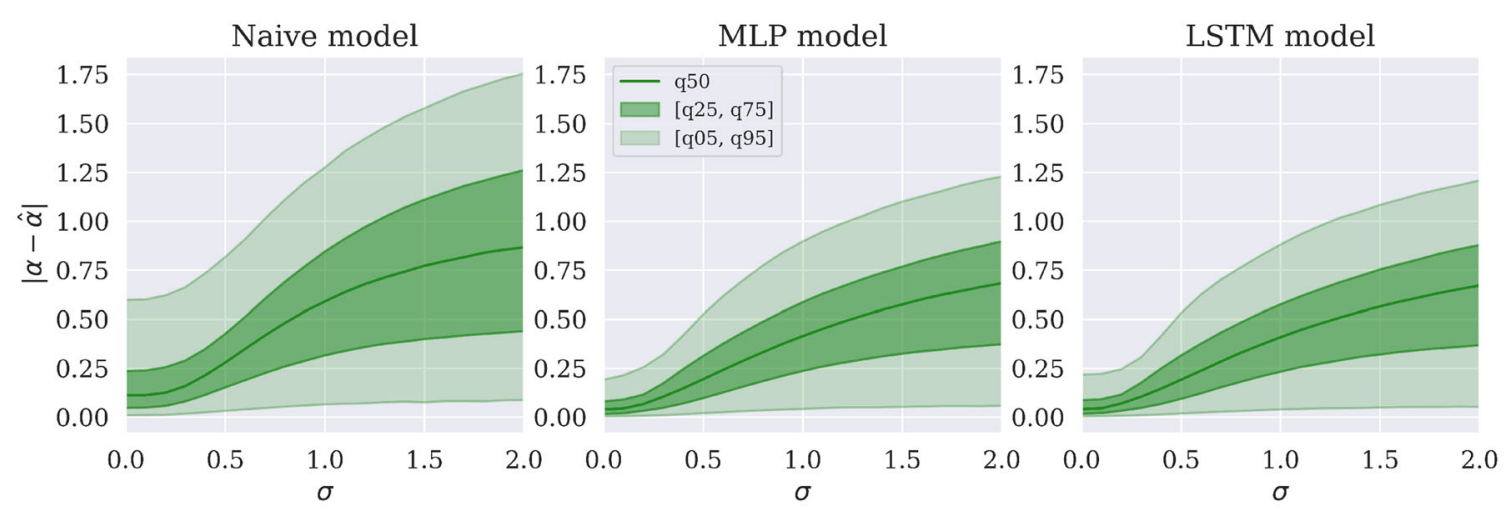

(a) MAE of $\alpha$ parameter estimating models for $\mathrm{fBm}$ anomalous diffusion process.
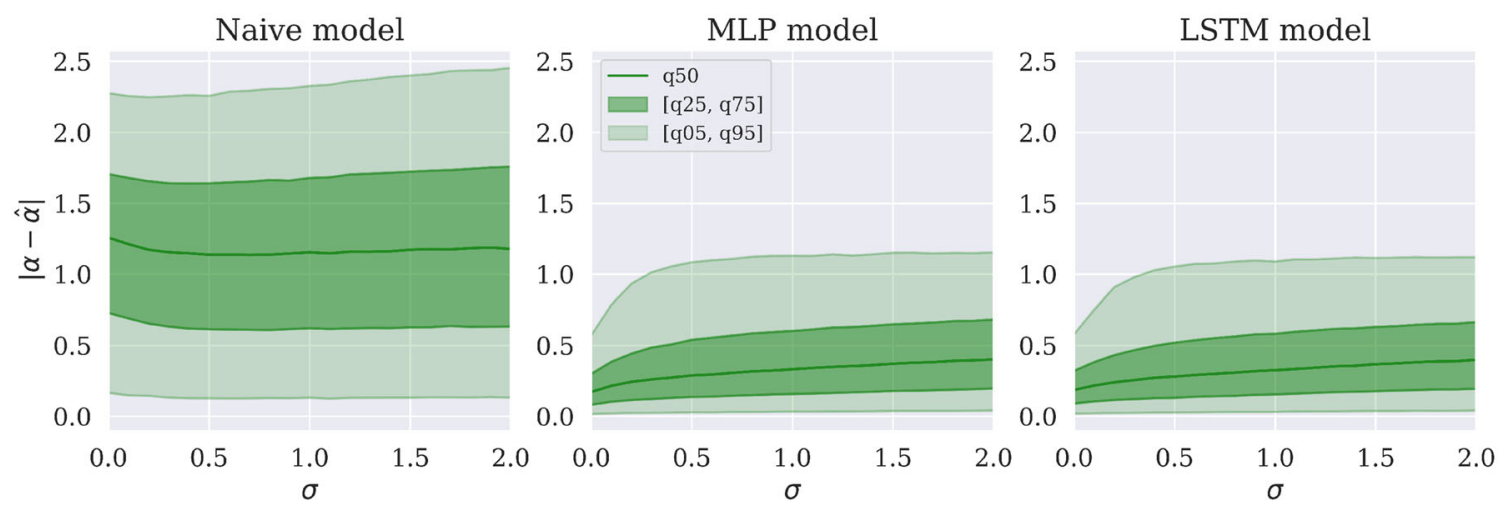

(b) MAE of $\alpha$ parameter estimating models for sBm anomalous diffusion process.

Fig. 5 Figures show the influence of noise on $\mathrm{MAE}$ for $\mathrm{fBm}$ and $\mathrm{sBm}$. Each plot contains quantile lines for different noise scales $\sigma \in[0,2]$

diffusion parameter with an accuracy that is more than sufficient to discern the diffusion type.

While in real-life applications the noise is omnipresent [31], it is critical for the estimators to withstand the influence of distortions and not degrade severely. For this reason, we validated and compared the theoretical estimators with NN-based ones, with and without dataset augmentation (which should be seen as a regularization technique [58]). The results show that $\mathrm{NN}$ approaches are more resilient to noise even without regularization. Additionally, with the added dataset augmentation, the robustness further improved and significantly surpassed the theoretical estimators. It was observed that this technique made $\mathrm{fBm}$ NN-based estimator significantly more robust; however, such effect was not as vivid for $\mathrm{sBm}$, by reason of independent increments.

The proposed NN models are of similar size (for easier comparison); however, it should be noted that better accuracy could be achieved with larger models. Here, we increased the size of the model (number of parameters) of the proposed in [34] MLP model and reached lower MAE. Thus, achieving even better results is within the reach of the hand (however, the larger the model, the more computation and time is needed to converge).

The LSTM layer at its time was revolutionary for natural language processing (NLP), wherein the problem of various input lengths is customary. However, in the famous paper (in the NLP community) "Attention is all you need" [59], the authors state that the mechanism of attention and transformer is superior to LSTM or other recurrent NN topologies, such as [60-62]. Thanks to the usage of layer normalization [63] and skip-connections (proposed in [64]), the NN can be extended to larger sizes without gradient related problems [44]. Furthermore, this architecture preserves the sense of the order of the vector as the LSTM architecture. In many domains, approaches based on the one presented in [65] have shown to be successful [66-69]. Thus, inspiration from this architecture (and other extensions [70-73]) could also lead to better results.

Another extension of the approach presented in this paper is to use other statistics than ACVF. Furthermore, the model can take as input more than one statistic and intelligently combine those to reach a more reliable estimator. What is more, for this application, other architectures of $\mathrm{NN}$ can perform better. In the literature, there exist several 
Fig. 6 Figures depict the influence of noise on MAE for $\mathrm{fBm}$ and $\mathrm{sBm}$ for retrained models with the inclusion in the dataset trajectories obscured by noise. Each plot contains quantile lines for different noise scales $\sigma \in[0,2]$

Fig. 7 In the figures, we present the effect of using augmented dataset on models' accuracy (measured with MAE) for $\mathrm{fBm}$ and $\mathrm{sBm}$. The comparison is made using quantile lines for different noise scales $\sigma \in[0,2]$
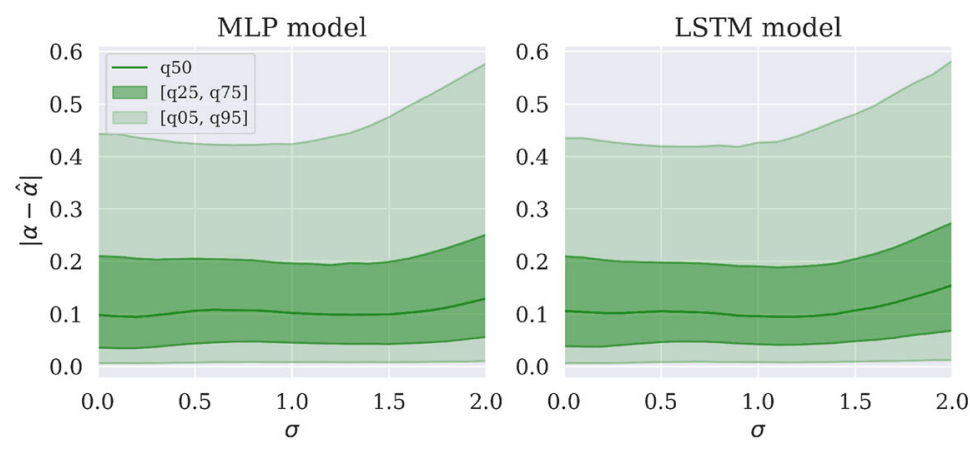

(a) MAE of $\alpha$ parameter estimating models for $\mathrm{fBm}$ anomalous diffusion process.
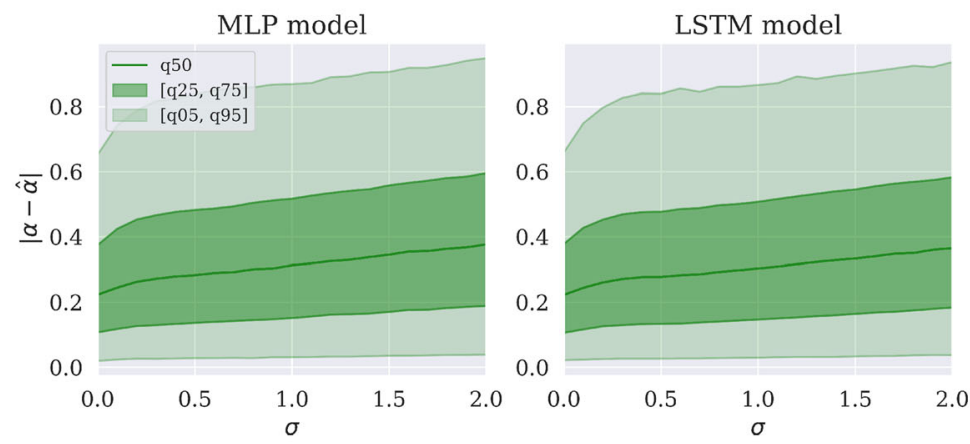

(b) MAE of $\alpha$ parameter estimating models for sBm anomalous diffusion process.
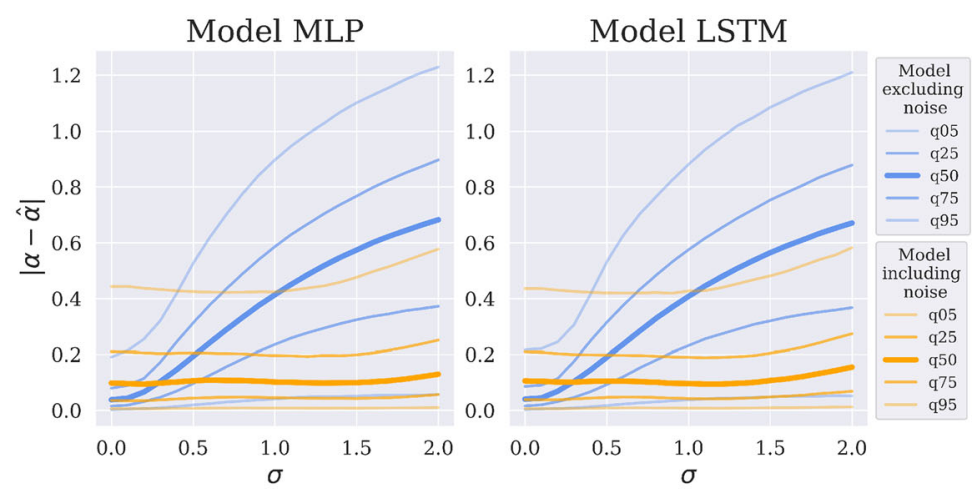

(a) MAE of $\alpha$ parameter estimating models for fBm anomalous diffusion process.
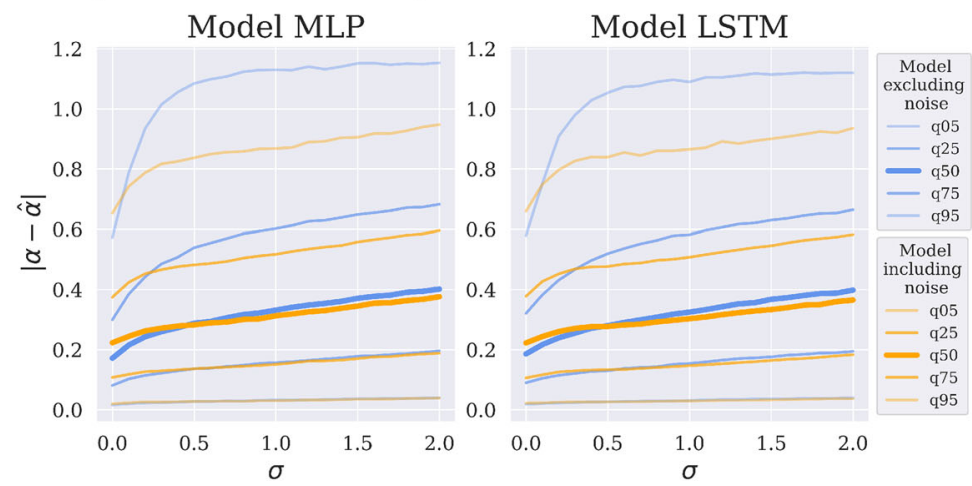

(b) MAE of $\alpha$ parameter estimating models for sBm anomalous diffusion process. 
other versions of recurrent layers [60-62]. Moreover, while we observed that the augmentation of the dataset helps in the models' generalization ability, thus other methods of regularization could help produce an estimator that is reliable also in other, more intricate, environments.

Funding This research received no external funding.

\section{Declarations}

Conflict of interest The author declares no conflict of interest.

Open Access This article is licensed under a Creative Commons Attribution 4.0 International License, which permits use, sharing, adaptation, distribution and reproduction in any medium or format, as long as you give appropriate credit to the original author(s) and the source, provide a link to the Creative Commons licence, and indicate if changes were made. The images or other third party material in this article are included in the article's Creative Commons licence, unless indicated otherwise in a credit line to the material. If material is not included in the article's Creative Commons licence and your intended use is not permitted by statutory regulation or exceeds the permitted use, you will need to obtain permission directly from the copyright holder. To view a copy of this licence, visit http://creativecommons.org/licenses/by/4.0/.

\section{References}

1. Jacobs, M.H.: Diffusion Processes. Ergebnisse der Biologie. Springer (1967)

2. Nezhadhaghighi, M.G., Rajabpour, M.A., Rouhani, S.: Firstpassage-time processes and subordinated Schramm-0-Loewner evolution. Phys. Rev. E 84, 011134 (2011)

3. Failla, R., Grigolini, P., Ignaccolo, M., Schwettmann, A.: Random growth of interfaces as a subordinated process. Phys. Rev. E 70, 010101(R) (2004)

4. Stanislavsky, A., Weron, K.: Two-time scale subordination in physical processes with long-term memory. Ann. Phys. 323(3), 643-653 (2008)

5. Gan, Y., Sun, L., Banhart, F.: One- and two-dimensional diffusion of metal atoms in graphene. Small 4(5), 587-591 (2008)

6. Lee, S.T., Gao, Z.Q., Hung, L.S.: Metal diffusion from electrodes in organic light-emitting diodes. Applied Physics Letters 75(10), 1404-1406 (1999)

7. Gabaix, X., Gopikrishnan, P., Plerou, V., Stanley, H.: A Theory of Power Law Distributions in Financial Market Fluctuations. Nature 423, 267-270 (2003)

8. Ivanov, P.C., Yuen, A., Podobnik, B., Lee, Y.: Common scaling patterns in intertrade times of US stocks. Phys. Rev. E 69, 05610 (2004)

9. Scher, H., Margolin, G., Metzler, R., Klafter, J.: The dynamical foundation of fractal stream chemistry: The origin of extremely long retention times. Geophysical Research Letters 29, 1061 (2002)

10. Doukhan, P., Oppenheim, G., (Eds.), M.S.T.: Theory and Applications of Long-Range Dependence. Birkhäuser Boston, Inc., Boston (2003)

11. Golding, I., Cox, E.C.: Physical nature of bacterial cytoplasm. Phys. Rev. Lett. 96, 098 (2006)

12. Muir, D.: Bulk flow and diffusion in the airways of the lung. Br. J. Dis. Chest 60(4), 169-176 (1966)
13. Mandelbrot, B.B., Van Ness, J.W.: Fractional Brownian Motions, Fractional Noises and Applications. SIAM Review 10(4), 422-437 (1968)

14. Stanislavsky, A., Burnecki, K., Magdziarz, M., Weron, A., Weron, K.: FARIMA modelling of solar flare activity from empirical time series of soft X-Ray Solar emission. Astrophys. J. 693, 1877-1882 (2009)

15. Smit, D.F.B.: Understanding molecular simulation: from algorithms to applications, 2nd edn, pp. 87-90. Computational science (San Diego, Calif.). Academic Press (2002)

16. Beran, J.: Statistics for Long-Memory Processes. Chapman \& Hall, pp. 55-59 (1994)

17. Thiel, F., Sokolov, I.: Scaled brownian motion as a mean-field model for continuous-time random walks. Phys. Rev. E Stat. Nonlinear Soft Matter Phys. 89, 012115 (2014)

18. Thiel, F., Sokolov, I.M.: Scaled Brownian motion as a mean-field model for continuous-time random walks. Phys. Rev. E 89, 012 (2014)

19. Safdari, H., Cherstvy, A.G., Chechkin, A.V., Bodrova, A., Metzler, R.: Aging underdamped scaled Brownian motion: Ensemble- and time-averaged particle displacements, nonergodicity, and the failure of the overdamping approximation. Phys. Rev. E 95, 012 (2017)

20. Samorodnitsky, G., Taqqu, M.: Stable Non-Gaussian Random Processes. Chapman \& Hall, New York, pp. 349-352 (1994)

21. Metzler, R., Klafter, J.: The random walk's guide to anomalous diffusion: a fractional dynamics approach. Phys. Rep. 339, 1-77 (2000)

22. Metzler, R., Klafter, J.: The restaurant at the end of the random walk: recent developments in the description of anomalous transport by fractional dynamics. J. Phys. A Gen. Phys. 37, R161-R208 (2004)

23. Sato, K.I.: Lévy Processes and Infinitely Divisible Distributions. Cambridge University Press, Cambridge (1999)

24. Wyłomańska, A.: Arithmetic Brownian motion subordinated by tempered stable and inverse tempered stable processes. Phys. A 391(22), 5685-5696 (2012)

25. Wyłomańska, A.: Tempered stable process with infinitely divisible inverse subordinators. J. Stat. Mech. Theory Exp. P10011 (2013)

26. Wyłomańska, A., Gajda, J.: Stable continuous-time autoregressive process driven by stable subordinator. Physica A 444, 1012-1026 (2016)

27. Magdziarz, M., Weron, A.: Fractional Fokker-Planck dynamics: stochastic representation and computer simulation. Phys. Rev. E 75, 056 (2007)

28. Magdziarz, M.: Langevin Picture of Subdiffusion with Infinitely Divisible Waiting Times. J. Stat. Phys. 135, 763-772 (2009)

29. Gajda, J., Magdziarz, M.: Fractional Fokker-Planck equation with tempered alpha-stable waiting times: Langevin picture and computer simulation. Phys. Rev. E 82, 011 (2010)

30. Gajda, J., Wyłomańska, A.: Fokker-Planck type equations associated with fractional Brownian motion controlled by infinitely divisible processes. Physica A 405, 104-113 (2014)

31. Maraj, K., Szarek, D., Sikora, G., Balcerek, M., Wyłomańska, A., Jabłoński, I.: Measurement instrumentation and selected signal processing techniques for anomalous diffusion analysis. Meas. Sens. 7-9, 100017 (2020)

32. Balcerek, M., Burnecki, K.: Testing of fractional Brownian motion in a noisy environment. Chaos Solitons Fractals 140, 110 (2020)

33. Balcerek, M., Burnecki, K., Sikora, G., Wyłomańska, A.: Discriminating gaussian processes via quadratic form statistics. Chaos An Interdiscip. J. Nonlinear Sci. 31(6), 063 (2021)

34. Szarek, D., Sikora, G., Balcerek, M., Jabłoński, I., Wyłomańska, A.: Fractional dynamics identification via intelligent unpacking 
of the sample autocovariance function by neural networks. Entropy 22(11) (2020)

35. Luiz Roberto Evangelista, E.K.L.: Fractional Diffusion Equations and Anomalous Diffusion, 1 edn. Cambridge University Press, Cambridge, pp. 86-116 (2018)

36. Hassler, U.: Stochastic Processes and Calculus: An Elementary Introduction with Applications. Springer Texts in Business and Economics. Springer (2015)

37. Garra, R., Issoglio, E., Taverna, G.S.: Fractional brownian motions ruled by nonlinear equations. Appl. Math. Lett. 102, 106160 (2020)

38. Nourdin, I.: Selected aspects of fractional Brownian motion. Bocconi \& Springer series 4. Springer, pp. 21-31 (2012)

39. Gut, A.: An Intermediate Course in Probability, 2 edn. Springer texts in statistics. Springer-Verlag New York, pp. 117-127 (2009)

40. Mitrinović, D.D.S.: Analytic Inequalities, 1 edn. Die Grundlehren der mathematischen Wissenschaften 165. Springer-Verlag Berlin Heidelberg, p. 109 (1970)

41. George Bachman, L.N.: Functional Analysis, 2nd edn, p. 29. Dover Books on Mathematics. Dover Publications (1998)

42. Hochreiter, S., Schmidhuber, J.: Long short-term memory. Neural Comput. 9(8), 1735-1780 (1997)

43. Gers, F.A., Schmidhuber, J., Cummins, F.: Learning to forget: continual prediction with LSTM. Neural Comput. 12, 2451-2471 (1999)

44. Pascanu, R., Mikolov, T., Bengio, Y.: Understanding the exploding gradient problem. arXiv preprint arXiv:1211.5063 (2012)

45. Bishop, C.M.: Neural Networks for Pattern Recognition. Oxford University Press, Oxford, pp. 116-161, 195-208 (1996)

46. Dekking, F., Kraaikamp, C., Lopuhaä, H., Meester, L.: A modern introduction to probability and statistics: understanding why and how. Springer Texts in Statistics. Springer, pp. 181-194 (2005)

47. Elfwing, S., Uchibe, E., Doya, K.: Sigmoid-weighted linear units for neural network function approximation in reinforcement learning. Neural Netw. 107, 3-11 (2018)

48. Ramachandran, P., Zoph, B., Le, Q.V.: Searching for activation functions. arXiv preprint arXiv:1710.05941 (2017)

49. Hendrycks, D., Gimpel, K.: Gaussian error linear units (GELUS). arXiv preprint arXiv:1606.08415 (2020)

50. Clevert, D., Unterthiner, T., Hochreiter, S.: Fast and accurate deep network learning by exponential linear units (elus). In: Bengio, Y., LeCun, Y. (eds.) 4th International Conference on Learning Representations, ICLR 2016, San Juan, Puerto Rico, May 2-4, 2016, Conference Track Proceedings (2016)

51. Maas, A.L., Hannun, A.Y., Ng, A.Y.: Rectifier nonlinearities improve neural network acoustic models. In: Proceedings ICML, vol. 30 , p. 3 (2013)

52. Kingma, D., Ba, J.: Adam: A method for stochastic optimization. In: International Conference on Learning Representations (2014)

53. Lample, G., Ballesteros, M., Subramanian, S., Kawakami, K., Dyer, C.: Neural architectures for named entity recognition. In: Proceedings of the 2016 Conference of the North American Chapter of the Association for Computational Linguistics: Human Language Technologies, pp. 260-270. Association for Computational Linguistics, San Diego, California (2016)

54. Sabour, S., Frosst, N., Hinton, G.: Dynamic routing between capsules. In: Guyon, I., Luxburg, U.V., Bengio, S., Wallach, H., Fergus, R., Vishwanathan, S., Garnett, R. (eds.) Advances in Neural Information Processing Systems 30, pp. 3856-3866. Curran Associates, Inc. (2017)

55. Dosovitskiy, A., Fischer, P., Ilg, E., Hausser, P., Hazirbas, C., Golkov, V., van der Smagt, P., Cremers, D., Brox, T.: Flownet: Learning optical flow with convolutional networks. In: 2015 IEEE International Conference on Computer Vision (ICCV), pp. 2758-2766 (2015)
56. Mao, X., Li, Q., Xie, H., Lau, R., Wang, Z., Smolley, S.P.: Least squares generative adversarial networks. In: Proceedings of the IEEE International Conference on Computer Vision (ICCV), pp. 2813-2821 (2017)

57. Cybenko, G.: Approximation by superpositions of a sigmoidal function. Math. Control Signals Syst. 2(4), 303-314 (1989)

58. Goodfellow, I., Bengio, Y., Courville, A.: Deep Learning. MIT Press, Cambridge, pp. 224-270 (2016)

59. Vaswani, A., Shazeer, N., Parmar, N., Uszkoreit, J., Jones, L., Gomez, A.N., Kaiser, L.u., Polosukhin, I.: Attention is all you need. In: Guyon, I., Luxburg, U.V., Bengio, S., Wallach, H., Fergus, R., Vishwanathan, S., Garnett, R. (eds.) Advances in Neural Information Processing Systems, vol. 30. Curran Associates, Inc. (2017)

60. Hoedt, P.J., Kratzert, F., Klotz, D., Halmich, C., Holzleitner, M., Nearing, G., Hochreiter, S., Klambauer, G.: MC-LSTM: massconserving LSTM. In: ICML (2021)

61. Neil, D., Pfeiffer, M., Liu, S.C.: Phased LSTM: accelerating recurrent network training for long or event-based sequences. In: Proceedings of the 30th International Conference on Neural Information Processing Systems, NIPS'16, pp. 3889-3897. Curran Associates Inc., Red Hook, NY, USA (2016)

62. Dey, R., Salem, F.M.: Gate-variants of gated recurrent unit (GRU) neural networks. In: 2017 IEEE 60th International Midwest Symposium on Circuits and Systems (MWSCAS), pp. 1597-1600 (2017)

63. Ba, J.L., Kiros, J.R., Hinton, G.E.: Layer normalization. arXiv preprint arXiv: 1607.06450 (2016)

64. He, K., Zhang, X., Ren, S., Sun, J.: Deep residual learning for image recognition. In: 2016 IEEE Conference on Computer Vision and Pattern Recognition (CVPR), pp. 770-778 (2016)

65. Devlin, J., Chang, M.W., Lee, K., Toutanova, K.: BERT: pretraining of deep bidirectional transformers for language understanding. In: Proceedings of the 2019 Conference of the North American Chapter of the Association for Computational Linguistics: Human Language Technologies, Volume 1 (Long and Short Papers. Association for Computational Linguistics, Minneapolis, Minnesota), pp. 4171-4186 (2019)

66. Dosovitskiy, A., Beyer, L., Kolesnikov, A., Weissenborn, D., Zhai, X., Unterthiner, T., Dehghani, M., Minderer, M., Heigold, G., Gelly, S., Uszkoreit, J., Houlsby, N.: An image is worth 16x16 words: transformers for image recognition at scale. arXiv preprint arXiv:2010.11929 (2021)

67. Wang, A., Singh, A., Michael, J., Hill, F., Levy, O., Bowman, S.: GLUE: A multi-task benchmark and analysis platform for natural language understanding. In: Proceedings of the 2018 EMNLP Workshop BlackboxNLP: Analyzing and Interpreting Neural Networks for NLP, pp. 353-355. Association for Computational Linguistics, Brussels, Belgium (2018)

68. Veličković, P., Cucurull, G., Casanova, A., Romero, A., Liò, P., Bengio, Y.: Graph attention networks. arXiv preprint arXiv:1710.10903 (2018)

69. Zhang, H., Goodfellow, I., Metaxas, D., Odena, A.: Self-attention generative adversarial networks. In: International Conference on Machine Learning, pp. 7354-7363. PMLR (2019)

70. Wu, Y., Lian, D., Gong, N.Z., Yin, L., Yin, M., Zhou, J., Yang, H.: Linear-time self attention with codeword histogram for efficient recommendation. In: Proceedings of the Web Conference 2021, WWW '21, p. 1262-1273. Association for Computing Machinery, New York, NY, USA (2021)

71. Thapak, P., Hore, P.: Transformer++. arXiv preprint arXiv:2003.04974 (2020)

72. Roy, A., Saffar, M., Vaswani, A., Grangier, D.: Efficient contentbased sparse attention with routing transformers. Trans. Assoc. Comput. Linguist. 9, 53-68 (2021) 
73. Fedus, W., Zoph, B., Shazeer, N.: Switch transformers: scaling to trillion parameter models with simple and efficient sparsity. arXiv preprint arXiv:2101.03961 (2021)
Publisher's Note Springer Nature remains neutral with regard to jurisdictional claims in published maps and institutional affiliations. 